\title{
O ensino da enfermagem gerontogeriátrica nas universidades federais brasileiras
}

Recebido em: 14/03/2012

Aceito em: 31/01/2013

\author{
Andréa Thayanara Santos Andrade' \\ Sheilla Paixão Silva Sampaio ${ }^{1}$ \\ Cristiane Franca Lisboa Gois ${ }^{2}$ \\ Maria Claudia Tavares de Mattos ${ }^{2}$ \\ Maria Pontes de Aguiar Campos ${ }^{2}$ \\ Gabryella Garibalde Santana Resende ${ }^{3}$ \\ Lincoln Vitor Santos 4
}

Objetivou-se identificar as características do ensino gerontogeriátrico ministrado pelos cursos de Enfermagem das IFES e averiguar as principais diferenças entre as regiões. Estudo quantitativo, realizado através de questionário e apurado pelo software SPSS. Os resultados mostram que os cursos de Enfermagem ministram o conteúdo isoladamente ou integrados a outras disciplinas, e apontam a necessidade de mudanças nos currículos para capacitar os profissionais para atender à população idosa.

Descritores: Educação em Enfermagem, Idoso, Geriatria.

\section{The teaching of geronto-geriatric nursing care at Brazilian federal universities}

This work aimed to identify the characteristics of the geronto-geriatric nursing care teaching in the Nursing courses of the IFES (Federal Higher Education Institutions) and assess the main differences between the regions. A quantitative study carried out through a questionnaire and assessed by SPSS. The results showed that the Nursing Courses approach such content separately or integrated to other subjects, and point out the need for changes in the curriculum framework in order to qualify the professionals to serve the older population.

Descriptors: Nursing Education, Elderly Person, Geriatrics.

\section{La enseñanza de la enfermería gerontogeriátrica en las universidades federales brasileñas}

Se buscó identificar las características de la enseñanza gerontogeriátrica ministrada por los cursos de Enfermería de las IFES y averiguar las principales diferencias entre las regiones. Estudio cuantitativo, llevado a cabo a través de cuestionario y levantado por el software SPSS. Los resultados muestran que los Cursos de Enfermería ministran el contenido aisladamente o integrados a otras disciplinas, y señalan la necesidad de cambios en los currículos para capacitar a los profesionales para atender a la población anciana.

Descriptores: Educación en Enfermería, Anciano, Geriatría.

\section{INTRODUÇÃO}

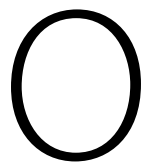
envelhecimento é um processo natural que ocorre ao longo da vida do ser humano. Trata-se de uma mudança na estrutura etária da população, sendo no Brasil considerada idosa a pessoa que apresenta 60 anos ou mais de idade ${ }^{(1)}$.

Nos últimos anos, houve um crescimento significativo da população idosa. Essa transição demográfica resulta não somente do declínio da fecundidade e mortalidade, como também da urbanização, do desenvolvimento da ciência e da institucionalização das aposentadorias ${ }^{(2)}$. No período de 1999 a 2009, a proporção de idosos na população do País passou de $9,1 \%$ para $11,3 \%{ }^{(3)}$.

Anualmente, somam-se $650 \mathrm{mil}$ idosos à população brasileira, cuja maioria possui doenças crônicas e algumas limitações funcionais ${ }^{(4)}$, como hipertensão arterial, diabetes, artrite e acidente vascular cerebral, que exigem cuidados constantes, medicação contínua e exames periódicos, entre outros $^{(5)}$.

Devido à necessidade de assistência integral ao idoso, a Associação Brasileira de Enfermagem (ABEn), juntamente com o Conselho Federal de Enfermagem (COFEN), Conselhos Regionais de Enfermagem (COREN) e Escolas de Enfermagem, apresentaram uma proposta curricular que contemplava a atenção à saúde do idoso de forma individual, em situações clínicas, cirúrgicas, gineco-obstétricas e psiquiátricas, em serviços hospitalares, não hospitalares e na comunidade ${ }^{(6)}$.

Através da Resolução CNE/CES n. ${ }^{\circ} 3$, de 7 de novembro de 2001, as Diretrizes Curriculares Nacionais do Curso de Graduação em Enfermagem, em seu artigo 50, estabelecem que o enfermeiro deve ser dotado de conhecimentos e 
habilidades específicas para "atuar profissionalmente, compreendendo a natureza humana em suas dimensões, em suas expressões e fases evolutivas" (7).

As disciplinas gerontologia e geriatria correspondem, respectivamente, ao conjunto de ciências, técnicas e saberes que têm como objeto de estudo o envelhecimento ${ }^{(8)}$ e a prevenção e tratamento das doenças decorrentes da terceira idade ${ }^{(9)}$. Ambas têm o intuito de capacitar o profissional para atender às demandas físicas, emocionais e socioculturais dessa população, melhorando a qualidade de vida e saúde do idoso e da sua família ${ }^{(10)}$.

Nesse sentido, as instituições de ensino devem preparar os futuros profissionais de saúde, em especial os enfermeiros, afim de conhecer a realidade social e de saúde do idoso focando na promoção da sua saúde, minimizar a dependência e potencializar a autonomia, favorecendo uma melhor qualidade de vida (11).

Visto que as instituições são responsáveis por formar profissionais, surgiu o interesse em identificar as características do ensino gerontogeriátrico ministrado aos acadêmicos dos cursos de Enfermagem das universidades federais brasileiras localizadas nas capitais, bem como averiguar as principais diferenças entre as regiões. Visa, ainda, contribuir para o entendimento da tendência pedagógica na formação do enfermeiro brasileiro e a adequação do perfil do profissional de enfermagem às reais necessidades da parcela idosa da sociedade.

\section{METODOLOGIA}

Trata-se de um estudo descritivo, exploratório, transversal, utilizando uma abordagem quantitativa, no qual se pretendeu identificar as características do ensino da Enfermagem Gerontogeriátrica nos cursos de Enfermagem das universidades federais brasileiras das capitais.

Os dados foram coletados através de um questionário composto de sete perguntas fechadas e três semiabertas, referentes ao conteúdo e metodologia adotada pelo curso de Enfermagem em relação ao ensino de Enfermagem Gerontogeriátrica. As questões foram adaptadas da pesquisa realizada por Santos ${ }^{(6)}$, em que foram questionadas a existência das disciplinas, ementário, natureza, presença de atividades práticas e complementares que contemplassem o ensino sobre o idoso. O mesmo foi enviado via online e via correios para 27 coordenadores/chefes dos cursos de Enfermagem das universidades federais brasileiras das capitais, das quais 17 responderam, duas recusaram-se a participar do estudo e as demais não se posicionaram.
Adotou-se como critério de inclusão a exigência de que os cursos de Enfermagem fossem sediados nas capitais e pertencentes a Instituições Federais de Ensino Superior, por entendermos que nestas as mudanças curriculares são mais rápidas.

Os dados obtidos foram processados pelo software Statistical Package for Social Sciences (SPSS) versão 20, através do qual foram gerados tabelas, gráficos e análise de dados a partir de parâmetros estatísticos.

$O$ pequeno número de estudos publicados sobre o ensino da Enfermagem Gerontogeriátrica, restringiu as discussões, levando, nesse sentido, a uma limitação do presente estudo.

\section{RESULTADOS E DISCUSSÕES}

Participaram do estudo $63 \%{ }^{(17)}$ dos cursos de Enfermagem das capitais brasileiras, vinculadas às universidades federais. Dentre os que responderam, $24 \%{ }^{(4)}$ têm nomeadamente a disciplina Enfermagem Gerontogeriátrica; $12 \%(2)$, Enfermagem Gerontológica; e 6\%(1), Enfermagem Geriátrica, conforme demonstrado na Tabela 1.

Chamou atenção o fato de $6 \%^{(1)}$ das escolas abordarem apenas o idoso doente, o que não é recomendado, pois as atuais mudanças detransiçãodemográficaseepidemiológicas devem trazer importantes consequências para a educação profissional em saúde. Isso porque as intervenções de saúde ao idoso devem extrapolar o antigo modelo biomédico, com enfoque apenas na doença, requerendo abordagens que contemplem aspectos biológicos, psicológicos, sociais e culturais (12).

As demais universidades, com exceção de uma localizada na região Sul, inserem o conteúdo em disciplinas distintas, tais como Enfermagem em Saúde do Adulto e Idoso, Enfermagem Clínica e Cirúrgica, Enfermagem na Saúde da Mulher, Cuidados Especiais nos Ciclos de Vida, Semiologia
Tabela 1 - Existência e Natureza das disciplinas Enfermagem Geriátrica, Gerontológica e Gerontogeriátrica, Aracaju/SE, 2012.

\begin{tabular}{|l|cc|cccc|}
\hline \multirow{2}{*}{ DISCIPLINAS } & $\begin{array}{c}\text { Existência } \\
\text { de disciplinas }\end{array}$ & \multicolumn{5}{c|}{ Natureza } \\
\cline { 2 - 6 } & Sim & $\%$ & Obrigatória & $\%$ & Optativa & $\%$ \\
\hline $\begin{array}{l}\text { Enfermagem } \\
\text { Geriátrica }\end{array}$ & 1 & 6 & 0 & 0 & 1 & 6 \\
$\begin{array}{l}\text { Enfermagem } \\
\text { Gerontológica }\end{array}$ & 2 & 12 & 0 & 0 & 2 & 12 \\
$\begin{array}{l}\text { Enfermagem } \\
\text { Gerontogeriátrica }\end{array}$ & 4 & 24 & 3 & 18 & 1 & 6 \\
Total & 7 & 41 & 3 & 18 & 4 & 24 \\
\hline
\end{tabular}

Fonte: Dados da pesquisa 
e Semiotécnica em Enfermagem, Urgência e Emergência e Saúde Coletiva.

Aesse respeito, a OrganizaçãoPan-Americana deSaúde(OPAS) recomenda, especialmente para os cursos de Enfermagem, que não sejam oferecidos conteúdos gerontogeriátricos parciais, integradosaoutras disciplinas, paranãosecorrerorisco de diluir ou reduzir esses conteúdos ao longo do curso ${ }^{(13)}$.

Dessa forma, a inclusão de disciplinas especialmente voltadas para a Enfermagem Gerontológica torna-se extremamente necessária nos currículos de graduação, com o objetivo de atender uma demanda em crescimento, pois uma deficiência na formação acadêmica dos enfermeiros trará como consequência um prejuízo de seu potencial assistencial.

Constatou-se que as universidades que oferecem exclusivamente a disciplina Enfermagem Gerontológica e/ou Geriátrica possuem uma carga horária média de 63 horas. Os demais cursos que diluem o conteúdo relativo ao idoso em outras disciplinas não disponibilizaram a carga horária voltada para o mesmo.

No que diz respeito à natureza da disciplina Enfermagem Gerontogeriátrica, esta é ofertada como obrigatória em $18 \%{ }^{(3)}$ das instituições. Porém, quando o conteúdo dessa disciplina é ministrado separadamente em Enfermagem Geriátrica e Gerontológica, essas assumem natureza optativa em $6 \%{ }^{(1)}$ e $12 \%{ }^{(2)}$, respectivamente, das universidades.

Esse achado é preocupante, visto que deixa a critério do aluno a opção pelo aprendizado voltado ao processo de envelhecimento e seus agravos. Nesse sentido, recomenda- se que as escolas de Enfermagem promovam alterações em seus currículos, com o objetivo de suprir as necessidades contemporâneas de saúde da população, formando profissionais críticos, capazes de atender demandas locais e regionais ${ }^{(14)}$.

Verificou-se que a ementa da disciplina Enfermagem Gerontogeriátrica tem como foco o estudo dos fundamentos de geriatria e gerontologia, os fenômenos biopsicossociais envolvidos no processo de envelhecimento dos seres humanos, a transição demográfica e epidemiológica, a importância do conhecimento dos instrumentos legais e a avaliação multidimensional na atenção à pessoa idosa, utilizando a sistematização da assistência.

Os cursos de Enfermagem que apresentam essa disciplina têm como objetivo oferecer conhecimento e espaço para reflexão crítica, discutir a política de atenção à pessoa idosa, os agravos que acometem essa população e sua extensão, analisar os meios disponíveis de atenção e sua adequada utilização pelo idoso, além de fornecer condição técnico-científica para melhorar o manejo com o idoso.

$\mathrm{Na}$ análise da variável que investiga a existência de "atividades práticas nas disciplinas que contemplam o ensino da enfermagem gerontogeriátrica", constatou-se que somente uma escola de Enfermagem, localizada na região Sul, não desenvolve atividades práticas, sendo que essas se encontram presentes em 94\% (16) dos cursos que participaram do estudo.

Tal achado demonstra um aspecto positivo na formação

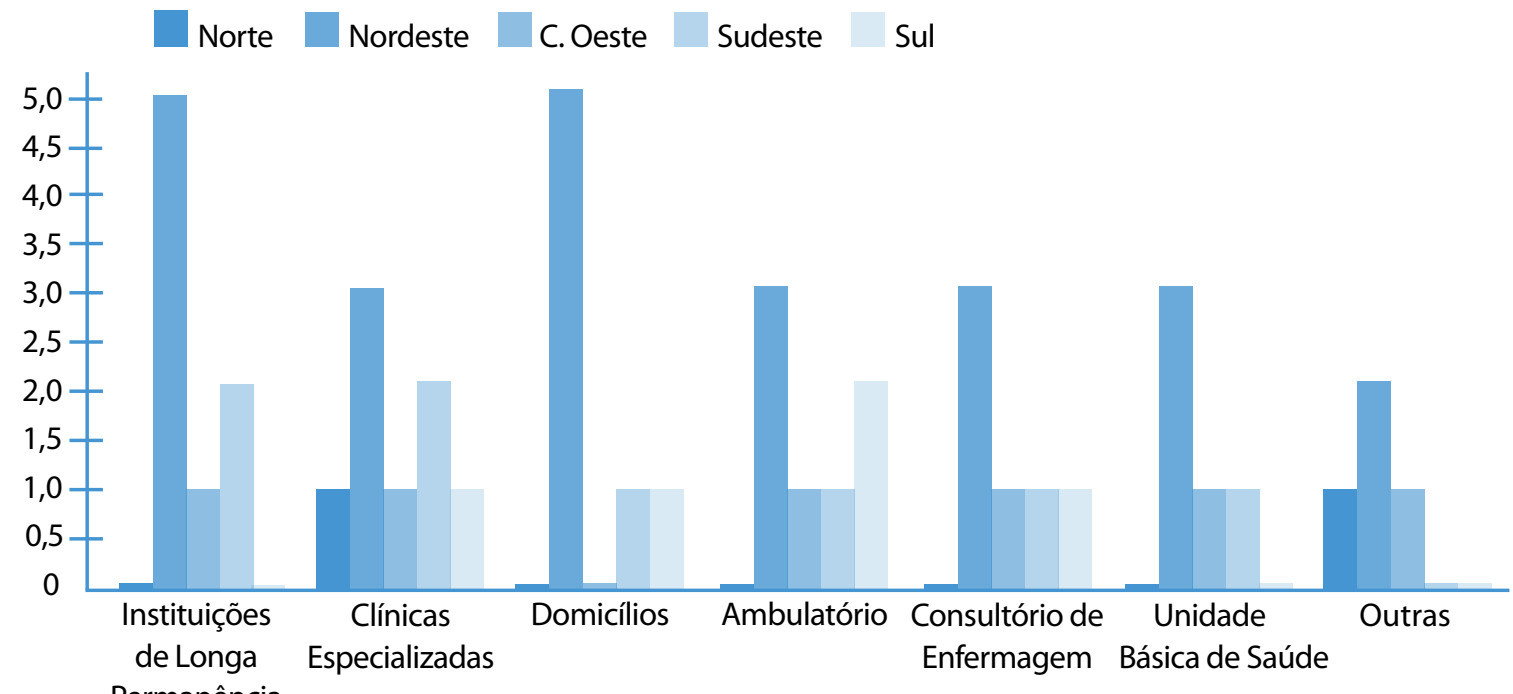

Figura 1 - Ocorrência de atividades práticas em gerontogeriatria, segundo as Regiões do país. Aracaju/SE, 2012. 
acadêmica, visto que as práticas auxiliam no planejamento e na prestação da assistência, na identificação das necessidades, na observação do cliente, e no estabelecimento de condutas para atender às necessidades do ser envelhecente ${ }^{(15)}$.

Com relação à investigação dos "locais selecionados para a realização das atividades práticas" pelos cursos de Enfermagem das Instituições Federais de Ensino Superior (IFES), foram citados por ordem de frequência: clínicas especializadas e em igual proporção, instituições de longa permanência, $47 \%{ }^{(8)}$; ambulatórios, $41 \%{ }^{(7)}$; domicílios, $41 \%{ }^{(7)}$; consultórios de enfermagem e unidades básicas de saúde, em igual percentual, $35 \%{ }^{(6)}$. Foram referidos outros campos de aulas práticas $12 \%{ }^{(2)}$, tais como centro de convivência de idosos e Universidade da Maturidade, com sede em uma capital na região Norte.

$\mathrm{Na}$ "análise das atividades complementares relacionadas à Enfermagem Gerontogeriátrica", pode-se observar que $81 \%^{(14)}$ das Universidades que compuseram a

"A maioria dos cursos de Enfermagem

não segue a preconização da OPAS no que se refere à inserção curricular de conteúdos gerontogeriátricos em disciplinas específicas"
Enfermagem das capitais da região Sudeste; $43 \%{ }^{(3)}$, da região Nordeste; e em $33 \%{ }^{(1)}$ dos cursos da região Sul do País. As regiões Centro-oeste e Norte não oferecem tais disciplinas.

Com relação à natureza curricular das disciplinas supracitadas, as mesmas se fazem obrigatórias em $33 \%^{(1)}$ das instituições pesquisadas da região Sudeste e em $29 \%{ }^{(2)}$ no Nordeste. Todas as escolas de Enfermagem da região Sul apresentam as disciplinas exclusivamente em caráter optativo.

No comparativo realizado entre as regiões com relação ao ementário das disciplinas citadas anteriormente, observou-se a existência de similaridade nos conteúdos que são abordados, dando-se um enfoque nas patologias mais comuns que acometem essa faixa etária, na Geriatria, no estudo dos fenômenos biopsicossociais e no processo de envelhecimento, na Gerontologia e na importância do conhecimento dos instrumentos legais em ambas.

No que diz respeito aos locais de realização das atividades práticas, a região Nordeste destaca-se em todos os campos, com maior participação em instituições de longa permanência e domicílios. A região Centro-Oeste apresenta uma proporção equivalente em todas as áreas, porém não atua em domicílios. O Sudeste mostra-se mais participativo em instituições de longa permanência e clínicas especializadas. A região Sul aparece como local de maior desenvolvimento de práticas nos ambulatórios, seguidos, em igual proporção, de clínicas especializadas, domicílios e consultórios de Enfermagem, enquanto que o Norte do País se mostra atuante apenas em clínicas especializadas e em outro campo, como a Universidade da Maturidade.

A região Nordeste destaca-se também como a região de mesmas encontram-se presentes em $100 \%{ }^{(3)}$ dos cursos de de alguma forma, a ausência de conteúdos teóricos nos currículos.

Quando se compara a existência das disciplinas Gerontológica e/ou Geriátrica entre as regiões brasileiras, as mesmas encontram-se presentes em $100 \%$ (3) dos cursos de

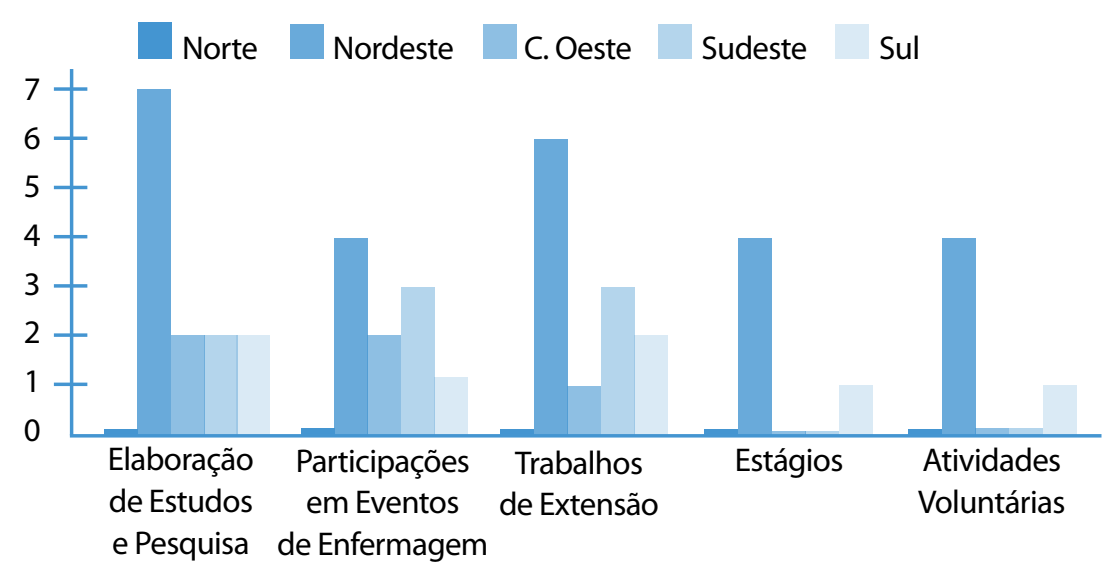

Figura 2 - Ocorrência de atividades complementares em gerontogeriatria, segundo as Regiões do País. Aracaju/SE, 2012. 
maior desenvolvimento de atividades complementares, conforme ilustrado na Figura 2, seguida das regiões Sudeste, Sul e Centro-oeste. A região Norte não realiza tais atividades.

Em relação a esse aspecto, nota-se que os dados encontrados são contraditórios com a realidade do País, visto que as regiões Sul/Sudeste apresentam as estruturas etárias mais envelhecidas, o que nos leva a constatar uma discordância dos cursos de Enfermagem das regiões Sul e Sudeste pesquisados quanto à preparação dos futuros profissionais de Enfermagem ${ }^{(16)}$.

\section{CONCLUSÃO}

A maioria dos cursos de Enfermagem não segue a preconização da OPAS no que se refere à inserção curricular de conteúdos gerontogeriátricos em disciplinas específicas.

A disciplina Enfermagem Gerontogeriátrica é obrigatória em $18 \%{ }^{(3)}$ das instituições. Porém, quando os conteúdos são ministrados separadamente, assumem natureza optativa em todos os cursos que participaram do estudo.

Quase a totalidade dos cursos de Enfermagem das IFES desenvolvem atividades práticas e complementares relacionadas à Enfermagem Gerontogeriátrica.

Espera-se, portanto, que em um futuro próximo todas as universidades brasileiras possam engendrar mudanças em seus currículos compatíveis com o crescimento demográfico significativo da população idosa, para que haja garantia de assistência de Enfermagem especializada e compatível a este grupo etário.

Sugere-se que outros estudos sejam realizados com foco na avaliação do ensino acerca do idoso nas Instituições de Ensino Superior, e que essas possam aderir à pesquisa, de modo a contribuir para a melhoria na formação dos futuros enfermeiros.

\section{Referências}

1. Ministério da Saúde (BR). Secretaria de Atenção à Saúde. Departamento de Açōes Programáticas e Estratégicas. Atenção à Saúde da Pessoa Idosa e Envelhecimento. Área Técnica de Saúde do Idoso [internet]. 2010. [citado em 2011 jul 6]. Disponível em: http://portal.saude.gov.br/portal/arquivos/pdf/volume12.pdf.

2. Silva LRF. Da velhice à terceira idade: o percurso histórico das identidades atreladas ao processo de envelhecimento. Hist Cienc Saúde. 2008;15(1):155-68.

3. Instituto Brasileiro de Geografia e Estatística. Síntese de Indicadores Sociais: uma análise das condições de vida da população brasileira [internet]. 2010. [citado em 2011 jul. 5]. Disponivel em: http://www.ibge.gov.br/home/estatistica/populacao/ condicaodevida/indicadoresminimos/sinteseindicsociais2010/SIS_2010.pdf

4. Veras R. Envelhecimento populacional e as informaçōes de saúde do PNAD: demandas e desafios contemporâneos. Introdução. Cad Saúde Pública. 2007;23(10):2463- 6 .

5. Giacomin KC, Peixoto SV, Uchoa E, Lima-Costa MF. Estudo de base populacional dos fatores associados à incapacidade funcional entre idosos na região Metropolitana de Belo Horizonte, Minas Gerais, Brasil. Cad Saúde Pública. 2008;24(6):1260-70.

6. Santos SSC. O ensino da enfermagem gerontogeriátrica e a complexidade. Rev Esc Enferm USP. 2006;40(2):228-35.

7. Brasil. Resolução da CNE/CES n 3, de 7 de novembro de 2001. Institui Diretrizes Curriculares Nacionais do Curso de Graduaçäo em Enfermagem [internet]. [citado 2011 jul. 7]. Disponível em: http://portal.mec.gov.br/cne/arquivos/pdf/CES03.pdf. 8. Pavarini SCI, Mendiondo MSZ, Barham EJ, Varoto VAG, Filizola CLA. A arte de cuidar do idoso: gerontologia como profissão? Texto Contexto Enferm. 2005;14(3):398-402.

9. Prado SD, Sayd JD. A gerontologia como campo do conhecimento científico: conceito, interesses e projeto político. Ciênc Saúde Coletiva. 2006;11(2):491-501.

10. Cardim MFM. A gerontologia como ferramenta de enfrentamento da mudança do perfil populacional. Rev Kairós. 2009 ago:71-82.

11. Martins JJ, Schier J, Erdmann AL, Albuquerque GL. Políticas públicas de atenção à saúde do idoso: reflexão acerca da capacitação dos profissionais da saúde para o cuidado com o idoso. Rev Bras Geriat Gerontol. 2007;10(3):371-82.

12. Mota $L B$, Aguiar $A C$. Novas competências profissionais em saúde e o envelhecimento populacional brasileiro: integridade, interdisciplinaridade e intersetorialidade. Ciênc Saúde Coletiva. 2007;12(2):363-72.

13. Diogo MJD. Formação de recursos humanos na área da saúde do idoso. Rev Latinoam Enferm. 2004;12(2):280-2.

14. Vasconcelos CMCB, Backes VMS, Gue JM. Avaliaçăo no ensino de graduação em enfermagem na America Latina: uma revisão integrativa. Enferm Global. 2011;23:118-39.

15. Diniz SB, Avelar MCQ. A prática docente de enfermeiros de instituiçōes de saúde numa Universidade privada. Ciênc Cuid Saúde. 2009;8(2):176-83.

16. Instituto Brasileiro de Geografia e Estatística. Pesquisa Nacional por Amostra de Domicílios - PNAD 2004. Composição e mobilidade populacional. [internet]. [citado em 2011 mai. 11]. Disponivel em: http://www.ibge.gov.br/mtexto/ pnadcoment1.htm. 